\title{
COMPARAÇÃO DE MÉTODOS DE CLASSIFICAÇÃO PARA O MAPEAMENTO DA COBERTURA DA TERRA NO SETOR NORTE DA ILHA DO MARANHÃO
}

\author{
CLASSIFICATION METHODS COMPARISON FOR THE MAPPING OF THE LAND COVER IN \\ THE NORTH SECTOR OF THE MARANHÃO ISLAND
}

\author{
Adilson Matheus Borges MACHADO, Taíssa Caroline Silva RODRIGUES \\ Pós-Graduação em Geoprocessamento, Especialização, Instituto Federal do Maranhão (IFMA). Avenida Colares Moreira, 477 - \\ Jardim Renascença, São Luís - MA. E-mails: adilsonborges94@gmail.com; taissageo@hotmail.com

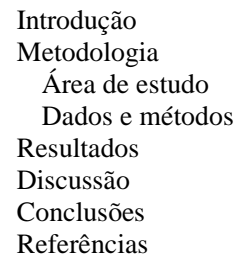

RESUMO - A zona costeira se estende das planícies costeiras até a borda externa das plataformas continentais. O método de classificação supervisionada consiste em um conhecimento prévio de algumas áreas a serem estudadas, o que permite amostras confiáveis. A GEOBIA (Geographic Object-Based Image Analysis) é um método eficaz na análise de imagens de alta resolução, incluindo fotointerpretação e classificação de características da paisagem. Assim, o presente estudo tem como objetivo principal comparar o método de classificação supervisionada pixel a pixel com o método de GEOBIA, buscando o mapeamento da cobertura da terra no setor Norte da Ilha do Maranhão. O algoritmo classificador utilizado foi o Maximum Likelihood Classification. O método de Classificação Supervisionada apresentou um valor de Kappa de 0,8469, Exatidão Global de 0,8950 e Variância Kappa de 0,0009295, a GEOBIA apresentou um valor de Kappa de 0,9410, Exatidão Global de 0,9600 e Variância Kappa de 0,0004075. O sensoriamento remoto mostra eficiência em pesquisas relacionadas aos usos e cobertura da terra, os resultados mostraram que ambos os métodos, apesar de suas limitações, mostraram resultados satisfatórios, o que torna interessante sua aplicação para iniciativas de mapeamento em diversos municípios brasileiros, em especial na zona costeira.

Palavras-chave: Classificação supervisionada. Mudanças ambientais. Cobertura da terra, GEOBIA.

\begin{abstract}
The coastal zone extends from the coastal plains to the external border of the coastal shelf. The GEOBIA (Geographic Object-Based Image Analysis) is an effective method for analyzing high-resolution images, including photointerpretation and classification of landscape features. The present study aims to compare the method of pixel to pixel supervised classification with the GEOBIA method for the mapping of the land cover in the North sector of the Maranhão Island. The supervised classification method consists of prior knowledge of some areas to be analyzed which allows the reliability of training samples. The classifier algorithm used was the Maximum Likelihood Classification. The Supervised Classification method showed a Kappa value of 0.8469, Global Accuracy of 0.8950 and Kappa Variance of 0.0009295. The GEOBIA presented a Kappa value of 0.9410, Global Accuracy of 0.9600 and Kappa Variance of 0.0004075 . Remote sensing proves to be extremely efficient in land use and occupation surveys. The results of the Supervised Classification method, despite their limitations, showed satisfactory results when compared to the results of the GEOBIA method, which makes interesting its application for mapping initiatives in several Brazilian municipalities, especially in the coastal zone.
\end{abstract}

Keywords: Supervised classification. Data mining. Environmental changes. Land cover, GEOBIA.

\section{INTRODUÇÃO}

A zona costeira tem seus limites definidos se estendendo das planícies costeiras até a borda externa das plataformas continentais, correspondendo aproximadamente à região que foi alternativamente inundada e exposta durante as flutuações do nível do mar, esta zona abriga uma combinação de ecossistemas de alta relevância ambiental, cuja diversidade é caracterizada pela transição entre ambientes terrestres e marinhos (Fabbri, 1998; Ver et al., 1999).

A obtenção de informações de imagens com o objetivo de realizar monitoramentos ambientais pode ser realizada com o auxílio de métodos de classificação automática de imagens ou interpretação visual. Para mapeamento do uso e cobertura da terra em cidades a interpretação visual em campo torna o processo mais caro, exige mais tempo e esforço dos pesquisadores especializados. A utilização de metodologias automáticas para a extrair informações é, portanto, uma opção viável para a classificação de uso e cobertura da terra em diferentes escalas espaciais e temporais (Pinho, 2005).

O Sensoriamento Remoto na perspectiva da paisagem e seus produtos, é uma ferramenta para a delimitação, classificação e avaliação das 
unidades paisagísticas. Nas últimas décadas o avanço das tecnologias de sistemas sensores e nas técnicas computacionais de processamento de imagens causou o refinamento da resolução espacial e na análise espacial das imagens e consequentemente dos estudos realizados (Jensen, 2009).

A Classificação Supervisionada de uma imagem de satélite multiespectral consiste em uma associação de cada pixel a uma "amostra" que descreve um objeto real (vegetação, área urbana, solo, etc.). Os valores numéricos associados a cada pixel são definidos pela reflectância dos materiais que os compõem, são identificados em termos de um tipo de cobertura da superfície terrestre imageada, denominadas de temas ou classes. As informações geradas sobre a cobertura da terra e uso de uma região, principalmente na zona costeira, são importantes para avaliação de mapeamento do desmatamento e aterro de manguezais, avaliação do risco de erosão, migração de dunas, identificação e análise da expansão urbana (Rwanga \& Ndambuki, 2017; Prudente et al., 2017).

O método de GEOBIA (Geographic ObjectBased Image Analysis) surgiu na década de 1990 como um método particularmente eficaz para análise de imagens de alta resolução espacial, incluindo fotointerpretação e classificação de características da paisagem. Na aplicação da GEOBIA, a classificação é então executada nos segmentos, em vez dos pixels tradicionais, levando em consideração as propriedades espectrais dos objetos como a textura, orientação, localização, topologia, brilho (Navulur, 2006; Kim et al., 2011; Rodrigues, 2014).

A GEOBIA oferece possibilidades onde as propriedades espectrais não são únicas, mas onde as relações entre a forma ou a vizinhança são distintas. Por exemplo, os meandros dos rios terão as propriedades espectrais da água quando ainda houver a circulação mas, uma vez abandonados, existe uma gama de possibilidades espectrais (Addink \& Kleinhans, 2008). Este método não se limita à comunidade de sensoriamento remoto, mas também abrange conceitos e princípios de Sistemas de Informação Geográfica (SIGs), ecologia de paisagem, entre outros (Blaschke et al., 2014).

Machado (2020) destaca a importância do método de Classificação de imagens para entender a complexidade das classes de uso e cobertura da Terra da Ilha do Maranhão, destacando que a resolução da imagem influencia diretamente nos resultados da classificação pixel a pixel. Rodrigues (2014) em seus estudos na ilha do Maranhão afirmou que a aplicação das técnicas de classificação da cobertura e uso da terra em áreas intraurbanas, trazem importantes conhecimentos que auxiliam o processo de planejamento urbano, ao fornecer subsídios para um diagnóstico detalhado das áreas de interesse.

Os requisitos de um monitoramento ambiental, das metas de conservação, aplicação do planejamento espacial ou gestão de recursos naturais orientada aos ecossistemas, impõem considerável urgência quanto ao desenvolvimento de soluções operacionais que podem extrair informações tangíveis de dados de sensoriamento remoto para diversas regiões. Assim o presente estudo tem o objetivo principal comparar o método de classificação supervisionada pixel a pixel com o método Geographic Object-Based Image Analysis (GEOBIA Classificação Orientada a Objeto) para o mapeamento da cobertura da terra no setor Norte da Ilha do Maranhão.

\section{METODOLOGIA}

\section{Área de estudo}

O Estado do Maranhão possui 217 municípios distribuídos em cinco regiões geográficas: Norte, o litoral; Oeste, ao longo dos grandes rios (Gurupi, Tocantins, Itinga e Araguaia); Sul, incluindo o Planalto das Mangabeiras; Centro, abrangendo o rio Mearim; e Leste, no curso do rio Parnaíba. O clima é tropical e existem 12 bacias hidrográficas, campos alagados, pântanos, savanas, palmeiras e florestas úmidas (IBGE 1997; De Matos et al., 2012; Feitosa \& Trovão, 2006).

A costa amazônica brasileira, se estende do norte do estado do Amapá até o Golfão Maranhense e representa 35\% de toda a costa brasileira, onde está inserida a Ilha do Maranhão, uma região caracterizada por uma variedade de ecossistemas estuarinos e marinhos diversos e pouco explorados (Couto et al., 2003; Sousa et al,. 2008; Ribeiro, et al., 2018). A região do Golfão Maranhense é influenciada por marés semidiurnas (ocorrência de duas baixa-mares e duas preamares por dia), com macromarés que podem chegar a 7 $\mathrm{m}$ de altura nas grandes sizígias (Marés Equinociais), com média de 6,6 m nas preamares, 
as correntes de maré podem chegar a $2,5 \mathrm{~m} \mathrm{~s}^{-1} \mathrm{e}$ alturas das ondas em torno de 0,6 a 1,4 m.

A região possui feições geomorfológicas características com extensas baixadas litorâneas com formações de praias arenosas e lodosas, dunas móveis, paleodunas, manguezais, marismas e uma extensa rede de canais, que são preenchidos a cada ciclo de preamar. Ao centro, sul e oeste, os manguezais, recortados por canais de maré, se relacionam à geomorfologia do Litoral Ocidental e do interior do Golfão Maranhense (Feitosa \& Trovão, 2006; El-Robrini et al., 2006).

A Ilha do Maranhão está localizada na feição geomorfológica do Golfão Maranhense e é constituída pelos municípios de São Luís (capital), São José de Ribamar, Paço do Lumiar e Raposa. O município de Raposa está localizado a norte da Ilha do Maranhão sendo limitado pelas coordenadas $02^{\circ} 21^{\prime} \mathrm{S}-02^{\circ} 32^{\prime} \mathrm{S}$ e $44^{\circ} 00^{\prime} \mathrm{W}-$ $44^{\circ} 12^{\prime}$ W (Figura 1) localizado a 28 km de São Luís (Feitosa, 1998; Feitosa \& Trovão, 2006; Piorski et al., 2009; Pereira et al., 2018). Segundo o Instituto Brasileiro de Geografia e Estatística IBGE (2010), o município da Raposa possui aproximadamente 26 mil habitantes distribuídos em 64 km² de extensão (Rangel, 2000).

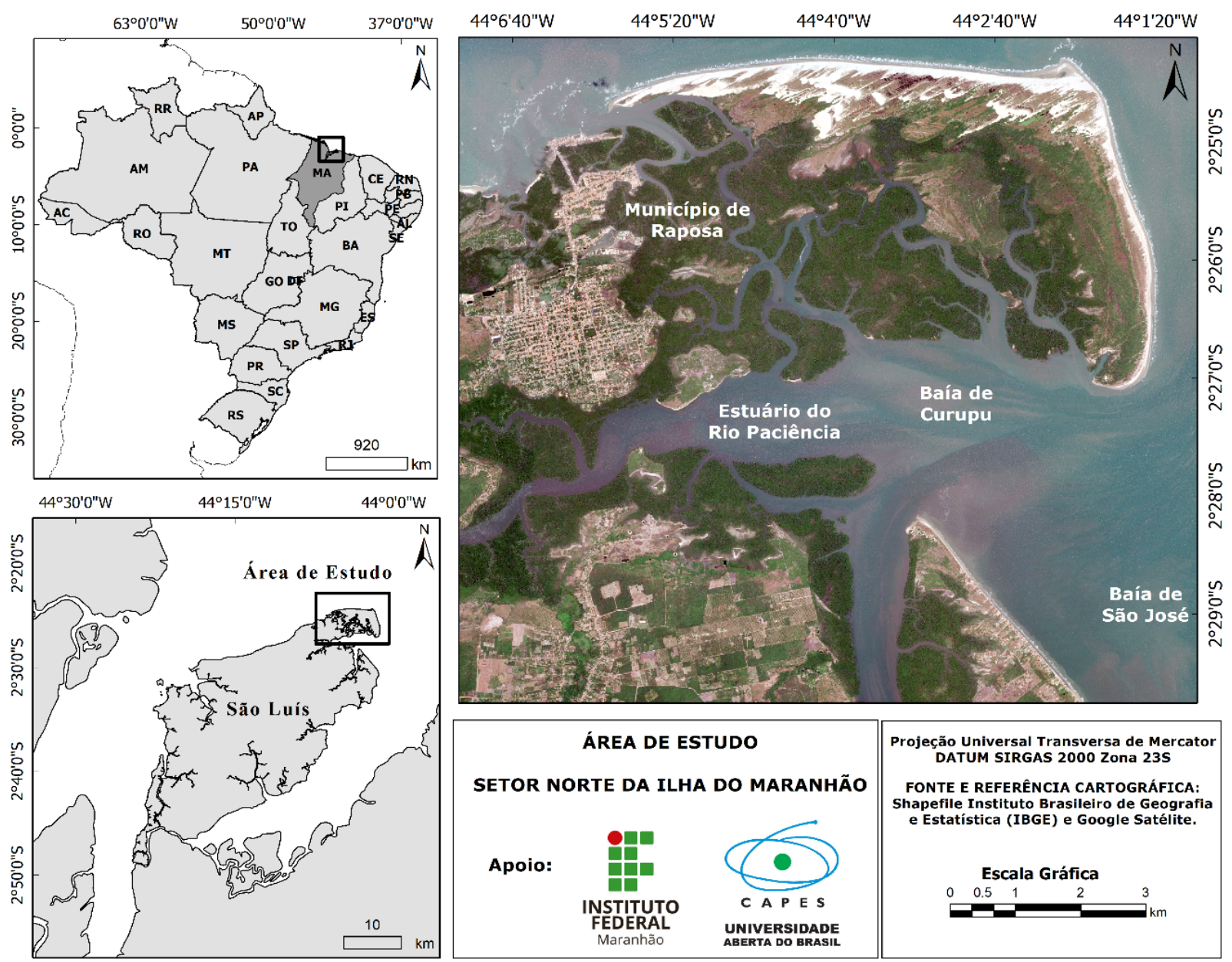

Figura 1 - Localização da Área de Estudo no setor Norte da Ilha do Maranhão.

No setor Norte da Ilha do Maranhão a vegetação predominante são os mangues. Os manguezais são ecossistemas costeiros, estuarinos, sujeitos a inundações periódicas pelas marés e por águas doces. São caracterizados pela entrada de sedimentos, água doce, nutrientes e saída de água e matéria orgânica para estuários e baías adjacentes, eles desempenham também um papel fundamental na ciclagem de nutrientes e abrigam uma fauna característica, são relativamente homogêneos e ocupam uma extensa faixa inundáveis pelas macromarés ao longo do litoral e nas embocaduras dos rios e igarapés (Cintrón \& Schaeffer-Novelli, 1992; Mochel \& Ponzoni, 2007). Os manguezais do município sofreram impactos constantes nos últimos anos por lançamentos de efluentes domésticos, resíduos sólidos bem como o desmatamento e aterramento para ocupação irregular e pela ação natural do deslocamento das dunas.

A região se torna ainda mais complexa pois o avanço da ocupação urbana exerce pressões sobre os diferentes ecossistemas da Zona Costeira da Ilha do Maranhão, a classificação 
desses ambientes é um aspecto relevante no levantamento de dados, os mapeamentos obtidos por meio desse método tornam a interpretação visual mais fácil e produzem importantes produtos que auxiliam no desenvolvimento de um planejamento urbano. Assim, as técnicas de classificação podem auxiliar na compreensão de impactos/características ambientais locais.

\section{Dados e métodos}

Nesta seção estão elencadas informações referentes aos dados e recursos para processamento e a metodologia utilizada para atingir os objetivos propostos, tais como: conjunto de imagens multiespectrais do satélite Landsat 8 sensor $O L I$ referente a 21/02/2016 em decorrência da cobertura de nuvens, com $30 \mathrm{~m}$ de resolução multiespectral e $15 \mathrm{~m}$ na banda pancromática obtidas no site do Serviço Geológico dos Estados Unidos (USGS) no endereço: http://earthexplorer.usgs.gov. As imagens foram georreferenciadas na projeção cartográfica Universal Transversa de Mercator; DATUM - SIRGAS 2000; zona 23 Hemisfério Sul.

Uma fusão das imagens foi realizada para combinar a informações espectrais das bandas multiespectrais com a informação geométrica (espacial), mais detalhada da banda pancromática (Wald et al., 1997; Novack, 2009; Rodrigues, 2014). Utilizando o no software QGIS 2.18.24 LTR inicialmente foram combinadas as oito bandas multiespectrais (resolução espacial $30 \mathrm{~m}$ ), com a banda pancromática (resolução espacial 15 $\mathrm{m})$, logo após, os recortes do limite estudado e classificação da cobertura da terra foram feitos.

A definição das classes de cobertura da terra foi realizada a partir da interpretação visual da imagem e a partir do conhecimento obtido da região, da análise do Plano Diretor das cidades e com base nos critérios de Florenzano (2002) e Jensen (2009). Após estes procedimentos, foram definidas 6 classes de cobertura da terra (água, bancos de areia, dunas/areia, mangues, vegetação rasteira e ocupação urbana) encontradas no setor Norte da Ilha do Maranhão com o auxílio do software ENVI 5.3, conforme consta na tabela 1, utilizando a combinações de bandas RGB 7,5,3 falsa cor para interpretação visual.

O software ENVI 5.3 foi utilizado também para transformação dos conjuntos de cenas em um único arquivo, para criar um mosaico das imagens e para a transformação para arquivos tiffs. O QGIS 2.18.24 LTR foi utilizado para criação de mapas temáticos. Já a aplicação da análise geográfica
Geographic Object-Based Image Analysis (GEOBIA) foram realizadas no software eCognition.

A classificação da cobertura da terra foi obtida em duas etapas, a saber: (1) definição das classes; (2) geração e análise dos atributos utilizados para a descrição das classes.

O método de classificação supervisionada consiste em um conhecimento prévio de algumas áreas nas quais se deseja trabalhar, o que permite amostras de treinamento confiáveis. O algoritmo classificador utilizado foi o Maximum Likelihood Classification ou máximo verossimilhança opera com base na distribuição de probabilidade de cada classe selecionada. Isto significa dizer que é um classificador paramétrico, ou seja, a distribuição dos valores de reflectância em uma área é descrita por uma função de densidade de probabilidade, desenvolvida com base na estatística Bayesiana. Este classificador avalia a probabilidade de um determinado pixel pertencer a uma mesma categoria e classifica o pixel para a categoria a qual ele tem a maior probabilidade de associação (Sun et al., 2013).

A análise da GEOBIA foi executada no software Ecogntion Developer 9.2. A primeira etapa foi o processo de segmentação que foi realizada utilizando o algoritmo multiresolution segmentation, para criação do tamanho apropriado dos objetos presentes na imagem, separando cada feição da paisagem. Para a realização da segmentação se utilizou como fator de escala 20 para uma melhor definição dos objetos em cena. Após esta etapa foi discriminada as classes que seriam identificadas na cena e assim foi coletado as amostras de cada uma delas para posterior exportação (Figura 2). A GEOBIA requer segmentação, atribuição, classificação e capacidade de consultar e vincular objetos individuais no espaço e no tempo.

Os dados foram exportados em forma de tabela o que deu a possibilidade da realização da mineração de dados no software WEKA gerando a árvore de decisão com o algoritmo J4.8. A rede semântica foi realizada pela aplicação de técnicas de mineração de dados, através da árvore de decisão, sendo ilustrada em formato de um fluxograma com a estrutura da árvore, facilmente convertido em regras de classificação (Han \& Kamber, 2006; Rodrigues, 2014). Dessa forma, a árvore de decisão foi inserida no software eCognition e a partir daí foi gerada a classificação final da cena. 
Tabela 1 - Descrição das classes de uso e cobertura da terra.

\begin{tabular}{|c|c|c|c|c|}
\hline CLASSE & AMOSTRA & $\begin{array}{c}\text { COR } \\
(\text { RGB753) }\end{array}$ & LOCALIZAÇÃO/CONTEXTO & $\begin{array}{c}\text { FORMA, TAMANHO } \\
\text { E } \\
\text { TEXTURA } \\
\end{array}$ \\
\hline Água & & $\begin{array}{l}\text { Cinza escuro a } \\
\text { preto. }\end{array}$ & $\begin{array}{l}\text { Áreas inundadas pela maré, água } \\
\text { marinha e/ou tanques de cultivos de } \\
\text { pescados. }\end{array}$ & $\begin{array}{l}\text { Textura lisa, formato e } \\
\text { tamanhos } \\
\text { variados. }\end{array}$ \\
\hline $\begin{array}{l}\text { Bancos de } \\
\text { Areia }\end{array}$ & & $\begin{array}{l}\text { Cinza escuro e } \\
\text { cinza claro }\end{array}$ & $\begin{array}{l}\text { Próximo à vegetação de mangue, } \\
\text { visíveis na baixa-mar. }\end{array}$ & $\begin{array}{l}\text { Forma alongada, } \\
\text { tamanho variável e } \\
\text { textura lisa e rugosa. }\end{array}$ \\
\hline $\begin{array}{c}\text { Dunas/Arei } \\
\text { a }\end{array}$ & & $\begin{array}{c}\text { Amarelo claro } \\
\text { ou } \\
\text { branco. }\end{array}$ & $\begin{array}{l}\text { Próximas à praia; em geral, possuem } \\
\text { vegetação ou mangue no entorno. }\end{array}$ & $\begin{array}{l}\text { Textura lisa, forma } \\
\text { irregular e } \\
\text { tamanhos variados. }\end{array}$ \\
\hline Mangues & & $\begin{array}{l}\text { Verde médio a } \\
\text { verde escuro. }\end{array}$ & $\begin{array}{l}\text { Localizado próximo } \\
\text { aos canais de maré. }\end{array}$ & $\begin{array}{c}\text { Extensa área de } \\
\text { vegetação com pouca } \\
\text { ou nenhuma presença } \\
\text { de telhados próximos. }\end{array}$ \\
\hline $\begin{array}{l}\text { Vegetação } \\
\text { Rasteira }\end{array}$ & & $\begin{array}{l}\text { Verde claro a } \\
\text { verde médio } \\
\text { com tons de } \\
\text { marrom. }\end{array}$ & $\begin{array}{c}\text { Dentro de quadras ou áreas } \\
\text { preservadas atrás dos mangues. }\end{array}$ & $\begin{array}{l}\text { Textura lisa a pouca } \\
\text { rugosa, forma e } \\
\text { tamanhos } \\
\text { variados. }\end{array}$ \\
\hline $\begin{array}{c}\text { Área } \\
\text { Urbanizada }\end{array}$ & & $\begin{array}{l}\text { Variações de } \\
\text { tons de } \\
\text { vermelho e } \\
\text { laranja. }\end{array}$ & $\begin{array}{l}\text { Dentro de quadras. Presença de } \\
\text { telhados aglomerados. }\end{array}$ & $\begin{array}{l}\text { Textura lisa a } \\
\text { levemente rugosa, } \\
\text { forma retangular e } \\
\text { tamanhos variados. }\end{array}$ \\
\hline
\end{tabular}

No software QGIS 2.18.24 LTR foram gerados 200 pontos amostrais espalhados de forma aleatória na imagem, para encontrar as referências terrestres. A avaliação dos pontos para se determinar a qual classe eles eram pertencentes, foi realizada por inspeção visual utilizando a combinações de bandas RGB 7,5,3 falsa cor. A partir da matriz de confusão (Congalton \& Green, 2009), foram calculados, o Índice Kappa e a Exatidão Global.

Por fim, para testar a significância estatística dos índices Kappa gerados nas duas classificações no setor Norte da ilha do Maranhão (com atributos 
espectrais e de texturais) (Francisco \& Almeida 2012b), foi utilizado o teste $\mathrm{Z}$ de acordo com a Equação 1 (Congalton \& Green, 2009). Sendo $K_{1}$ e $\mathrm{K}_{2}$ os índices Kappa da Classificação Supervisionada e da GEOBIA, respectivamente, e $\sigma_{1}^{2}$ e $\sigma_{2}^{2}$ a variância dos índices Kappa da Classificação Supervisionada e da GEOBIA, respectivamente. $\mathrm{O}$ teste $\mathrm{Z}$ para as os índices
Kappa das classificações foi realizado a 95\% de significância. Quando Z for $>1,96$, o teste é significativo, e rejeita-se a hipótese de nulidade, podendo concluir que existe diferença estatística entre os valores calculados (Vilela et al., 2005).

$$
Z=\frac{K_{1}-K_{2}}{\sqrt{\sigma_{1}^{2}+\sigma_{2}^{2}}}
$$
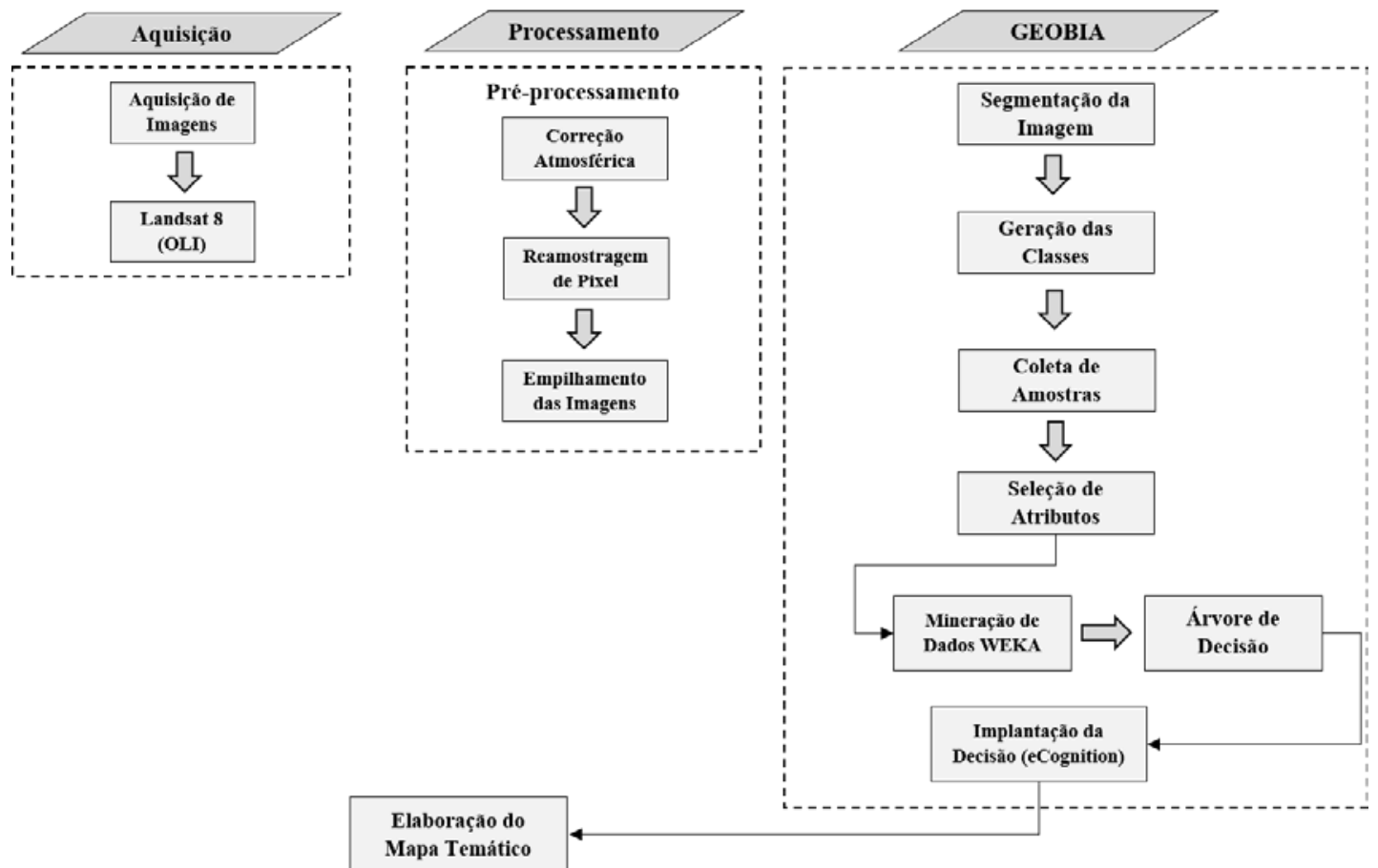

Figura 2 - Fluxograma da metodologia utilizada para geração do mapa temático de GEOBIA.

\section{RESULTADOS}

Foi aplicada a classificação supervisionada (Figura 3) e a GEOBIA (Figura 5) com um total de 6 classes cada um, já apresentados na tabela 1 . A verificação dos erros provenientes do processo de Classificação Supervisionada comparando com o método de GEOBIA obtidos pelo mapeamento temático foram realizadas utilizando coeficientes extraídos das matrizes de confusão. A partir dos resultados, Landis \& Koch (1977) associam valores do Índice Kappa à qualidade da classificação de acordo com a tabela 2.

A tabela 3 apresenta os valores de Kappa, Exatidão Global e Variância Kappa tanto para a Classificação Supervisionada quanto para GEOBIA, o método de Classificação Supervisionada apresentou um valor de Kappa de 0,8469, Exatidão Global de 89\% e Variância Kappa de 0,0009295, já o método de GEOBIA apresentou um valor de Kappa de 0,9410, Exatidão Global de 0,9600 e Variância Kappa de 0,0004075 .
Tabela 2 - Qualidade da classificação associada aos valores do Índice Kappa.

\begin{tabular}{c|c}
\hline Índice Kappa & Qualidade \\
\hline$<\mathbf{0 , 0}$ & Péssima \\
\hline $\mathbf{0 , 0 1}<\mathbf{K} \leq \mathbf{0 , 2 0}$ & Ruim \\
\hline $\mathbf{0 , 2 1}<\mathbf{K} \leq \mathbf{0 , 4 0}$ & Razoável \\
\hline $\mathbf{0 , 4 1}<\mathbf{K} \leq \mathbf{0 , 6 0}$ & Boa \\
\hline $\mathbf{0 , 6 1}<\mathbf{K} \leq \mathbf{0 , 8 0}$ & Muito Boa \\
\hline $\mathbf{0 , 8 1}<\mathbf{K} \leq \mathbf{1 , 0 0}$ & Excelente \\
\hline
\end{tabular}

Tabela 3 - Estatísticas geradas para as classificações de cobertura da terra.

\begin{tabular}{c|c|c|c}
\hline Método & Kappa & $\begin{array}{c}\text { Exatidão } \\
\text { global (\%) }\end{array}$ & $\begin{array}{c}\text { Variância - } \\
\text { kappa }\end{array}$ \\
\hline $\begin{array}{c}\text { Classificação } \\
\text { Supervisionada }\end{array}$ & 0,8469 & $89 \%$ & 0,0009295 \\
\hline GEOBIA & 0,9410 & $96 \%$ & 0,0004075 \\
\hline
\end{tabular}

Todas as classes ficaram com índice de exatidão global acima de $50 \%$, este resultado mostrou que o índice foi excelente. Apesar dos dois métodos apresentarem valores elevados, 
tanto no Índice Kappa como na Exatidão Global, conforme a tabela 3 , o resultado obtido por meio da GEOBIA apresentou valores comparativamente mais elevados.

$\mathrm{O}$ resultado do teste $\mathrm{Z}$ oriundo da comparação entre o método da GEOBIA e o método da Classificação Supervisionada apresentou o valor de 1,02 e valor de $p$ de 0,1533 , como $\mathrm{Z}$ foi $<1,96$ o teste não é significativo, portanto não existe diferença estatística entre os valores calculados para os dois métodos.

Na análise de Classificação Supervisionada observaram-se os impactos referentes a expansão urbana desordenada, além de ocupações irregulares em áreas de manguezais (Figura 3).
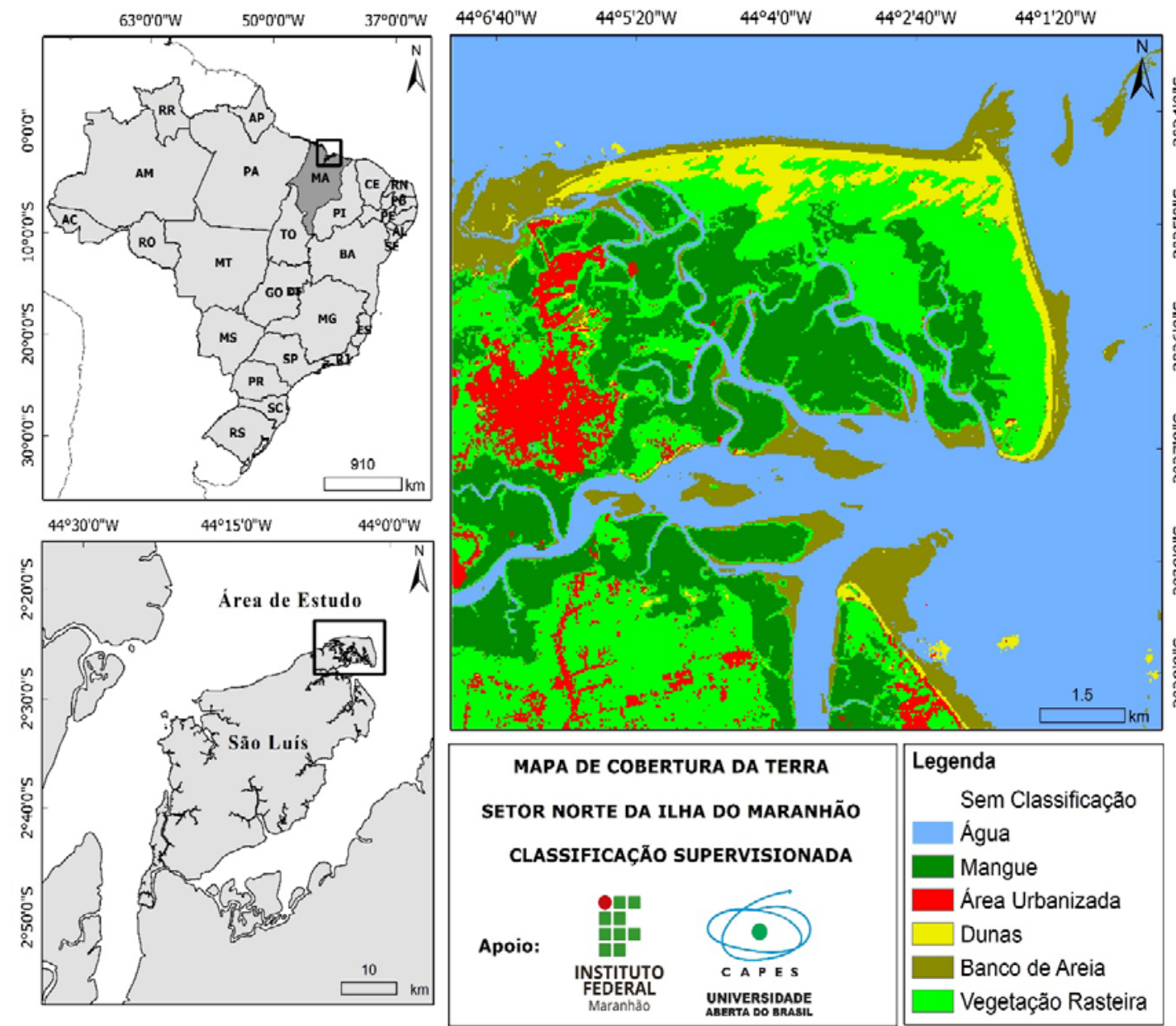

Figura 3 - Mapa da análise de Classificação Supervisionada no setor Norte da Ilha do Maranhão.

Após a etapa de mineração de dados, o banco de dados contendo os atributos espaciais e espectrais extraídos dos objetos (segmentos) da imagem Landsat 8, sensor OLI foi utilizado. A árvore de decisão com os atributos considerados para classificação pelo GEOBIA e as regras de decisão considerados são apresentados na figura 4, para a realização deste estudo, atributos como textura, a média da resposta espectral de cada banda, o desvio padrão, o índice de vegetação (NDVI) e o índice de umidade (NDWI), foram muito utilizados.

O método Gray-Level Co-occurrence Matrix (GLCM) contém informações sobre as características texturais da imagem levando em consideração a posição específica de um pixel em relação ao outro, que depende da construção de matrizes pela contagem do número de ocorrências de pares de pixels de determinadas intensidades em um determinado deslocamento, ou seja, todos os índices de textura GLCM possuem uma entrada que corresponde ao número de ocorrências espacial de tons de cinza dos vizinhos angulares próximos (Haralick et al., 1973).

Inicialmente, a árvore de decisão divide-se em dois grandes ramos. No ramo da esquerda, foram classificadas as classes com índice menor, como: água, mangue, área urbanizada e bancos de areia. No ramo da direita, que possui as classes com os maiores índices, foram classificadas utilizando o NDVI, o NDWI, a textura e a média da resposta espectral, as classes de vegetação rasteira e mangue.

As últimas componentes do ramo direito 
mostram o índice de umidade sendo determinante para diferenciação das classes de Vegetação Rasteira e Área Urbanizada.
As duas classificações apresentaram classes de uso da terra iguais, sendo uma maior distinção entre as classes na análise de GEOBIA (Figura 5).

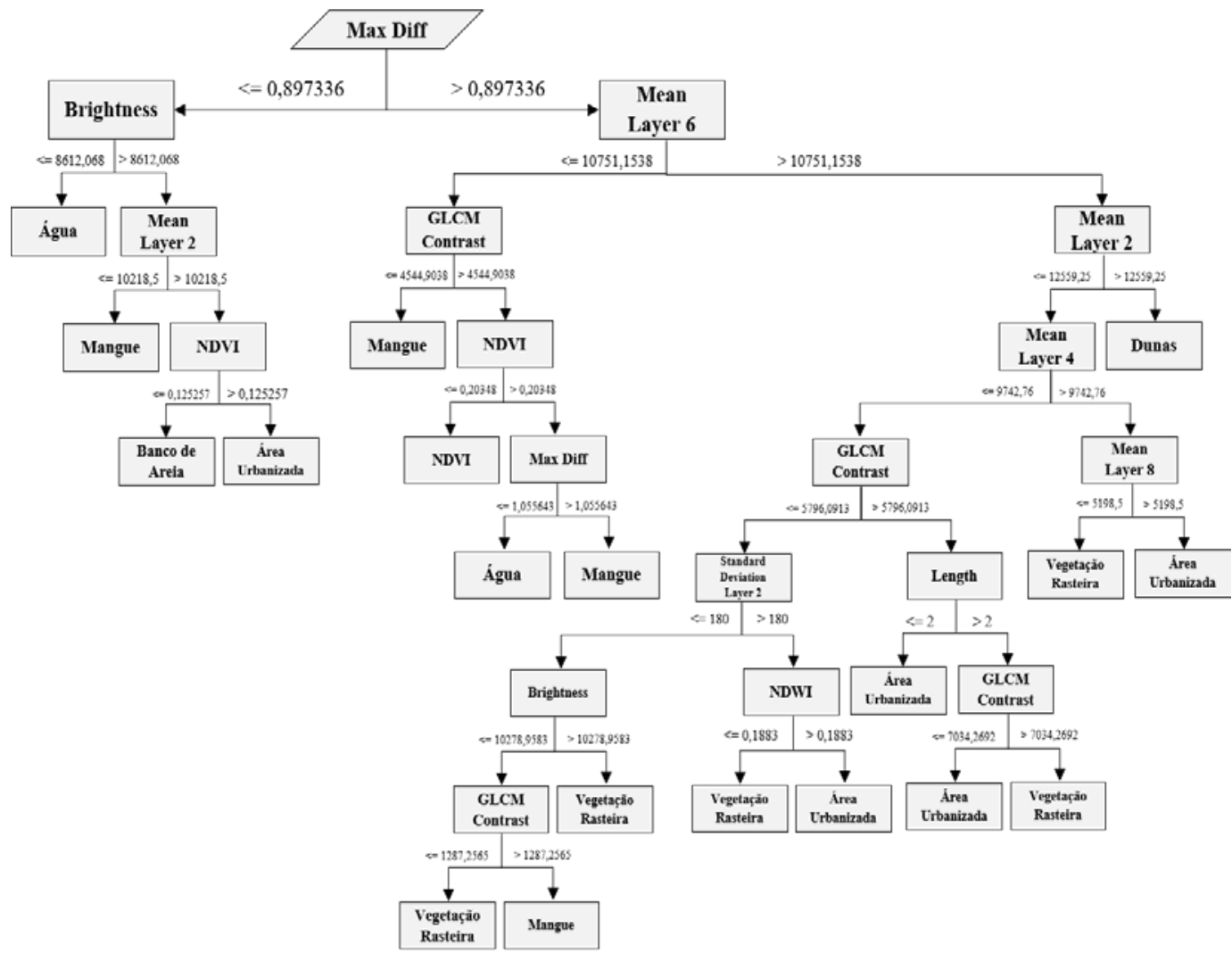

Figura 4 - A árvore de decisão, considerando a classificação pelo método de GEOBIA.
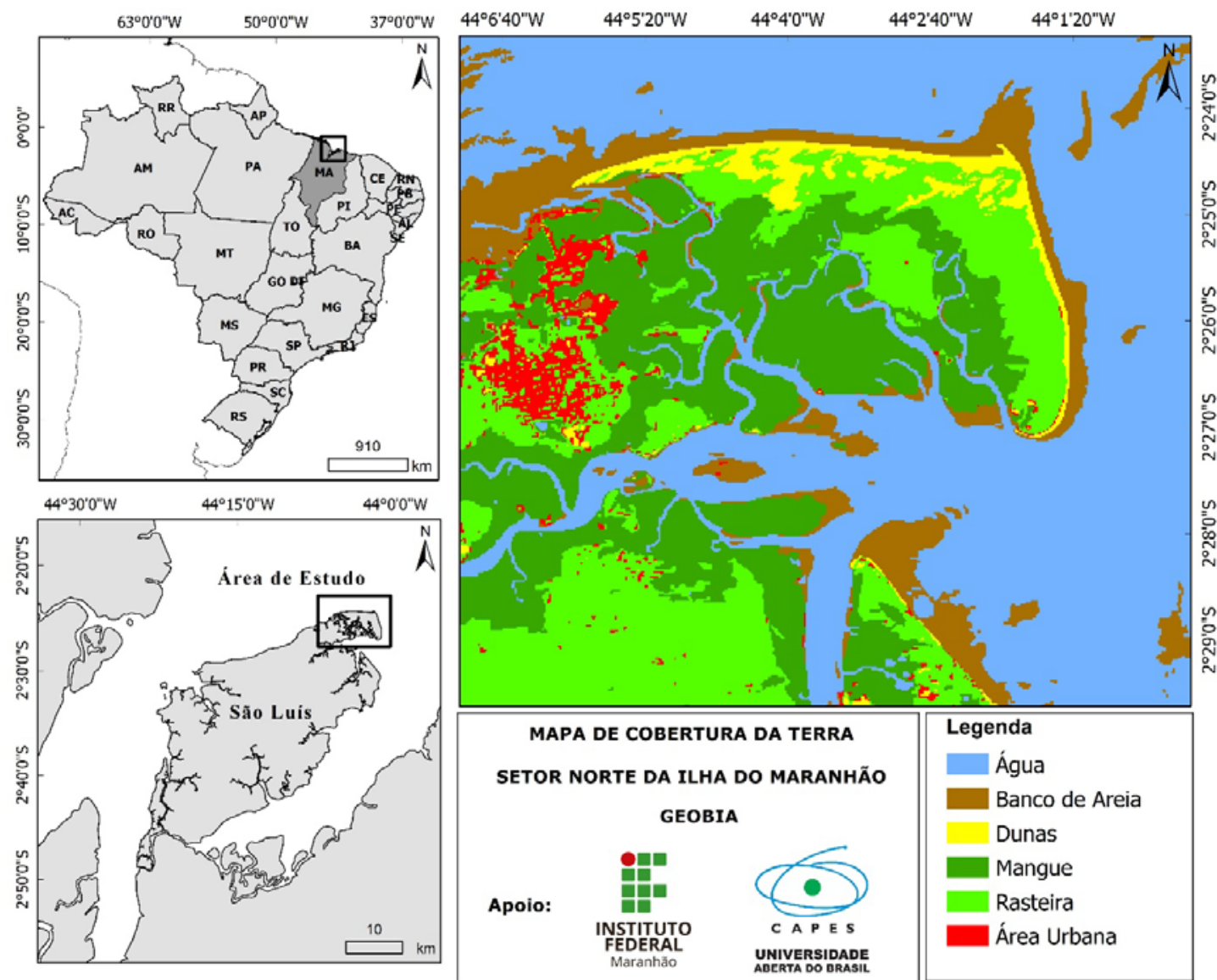

Figura 5 - Mapa da análise de GEOBIA no setor Norte da Ilha do Maranhão. 
A análise de GEOBIA, apresentou setores maiores da vegetação rasteira, principalmente na porção sul da área de estudo, quando comparado a análise de Classificação Supervisionada, que nesta mesma região apresentou maior frequência da classe “Área Urbanizada” (Figura 3).

A tabela 5 mostra os índices Kappa condicional para cada classe, Classificação Supervisionada e GEOBIA.

Para a Classificação Supervisionada a classe que apresentou o valor máximo do índice Kappa foi a de vegetação rasteira $(0,9272)$ e a que apresentou o menor valor foi a classe Dunas $(0,3232)$. Para o método de GEOBIA a tabela 5 mostra que a classe que apresentou o valor máximo do índice Kappa foi a de "Dunas" e "Banco de Areia" (1,000), representando perfeitamente as classes quando comparadas a natureza e a que apresentou o menor valor foi a classe de “Área Urbanizada” (0,8096), que no setor Sul da Área de Estudo não conseguiu distinguir muito bem as classes.

Tabela 4 - Gráfico dos índices Kappa - condicional e Qualidade para o método da Classificação Supervisionada e da GEOBIA para as classes de cobertura da terra do setor Norte da Ilha do Maranhão.

\begin{tabular}{c|c|c|c|c}
\hline CLASSES & $\begin{array}{c}\text { Classificação } \\
\text { Supervisionada Índice } \\
\text { kappa }\end{array}$ & $\begin{array}{c}\text { Qualidade da } \\
\text { Classificação } \\
\text { Supervisionada }\end{array}$ & $\begin{array}{c}\text { GEOBIA } \\
\text { Índice kappa }\end{array}$ & $\begin{array}{c}\text { Qualidade da } \\
\text { GEOBIA }\end{array}$ \\
\hline Água & 0,9038 & Excelente & 0,9802 & Excelente \\
\hline Mangue & 0,7471 & Muito Boa & 0,9011 & Excelente \\
\hline Área Urbanizada & 0,8076 & Muito Boa & 0,8096 & Muito Boa \\
\hline Duna & 0,3232 & Razoável & 1,0000 & Excelente \\
\hline Banco de Areia & 0,8430 & Excelente & 1,0000 & Excelente \\
\hline Vegetação Rasteira & 0,9272 & Excelente & 0,9295 & Excelente \\
\hline
\end{tabular}

\section{DISCUSSÃO}

Na Classificação Supervisionada as classes “Água” e "Vegetação Rasteira” apresentaram classificação satisfatória, principalmente as áreas desflorestadas devido às áreas destinadas a implementação de infraestrutura como construção de estradas. A classe "Banco de Areia”, apresentou confusão com a classe "Dunas”, apresentando um desempenho insatisfatório neste método. Essa confusão foi percebida nos dois métodos de classificação devido a complexidade em se classificar ambientes costeiros. Além disso a maré encontrava-se em baixa-mar no momento da passagem do satélite, o que aumentou a confusão entre essas duas classes.

Os resultados mostram que a Classificação Supervisionada e a análise do resultado da classificação orientada a objetos (GEOBIA) apresentaram resultados similares na busca pela representação das classes de uso e cobertura da terra. A área de estudo é de alta complexidade devido ao seu regime de marés que é semidiurno e de macromarés que atingem altura de 7 metros (nas marés Equinociais) a separação das classes é dificultada, causando o erro de classificação, pois a classe "Banco de Areia" que fica na interface continente-oceano, apresenta uma resposta espectral diferente da classe "Dunas" devido a esta classe ser influenciada pela subida e descida das marés, deixando os bancos úmidos (El-Robrini et al., 2006).

A eficiência da GEOBIA está no método que parte do princípio de que os objetos fornecem uma base de dados ampla para a classificação, ou seja, uma quantidade maior de atributos é levada em consideração e não apenas a resposta espectral dos alvos (Marpu, 2009). O método corresponde a um agrupamento de pixels contíguos, sendo possível calcular, parâmetros estatísticos e outros coeficientes relacionados às suas propriedades pixel a pixel (Francisco \& Almeida, 2012b). Outra vantagem do uso da GEOBIA em relação a outros métodos de classificação é a possibilidade de incorporação de dados temáticos e vetoriais no processo de modelagem das classes, sendo possível integrar conhecimentos prévios das mais diversas fontes para a modelagem de classes de maior complexidade.

A árvore de decisão discrimina os dois tipos de vegetação, Mangue e Vegetação Rasteira, uma vez que é normal a ocorrência de confusão entre essas classes, a textura e a umidade foram determinantes para a classificação dos Manguezais da região. A rede semântica elaborada por meio de mineração de dados demonstra precisão, a automatização dos procedimentos de seleção de 
atributos e definição das regras de decisão mostram potencial para regiões de alta complexidade que necessitam de uma interpretação visual mais precisa (Francisco \& Almeida, 2012a).

$\mathrm{Na}$ elaboração de mapas temáticos de cobertura da terra, nem sempre o resultado traduz a realidade com exatidão. No método de GEOBIA o menor valor do índice Kappa foi atribuído a classe "Área Urbanizada” que em certos locais não representou tão bem a ocorrência de urbanização, principalmente no setor sul da área de estudo. A confusão encontrada entre algumas classes, ainda que tenham sido quantitativamente bem reduzidos, apontam para a necessidade de teste com outros atributos e um número maior de amostras coletadas.

De maneira geral, a tabela 4 mostrou que ambas as classificações realizadas apresentaram resultados satisfatórios, sendo discriminadas todas as classes propostas. A separação entre as classes também foi avaliada por meio do índice Kappa - condicional. Observa-se que os valores destes índices são altos (próximos de 1), indicando um bom nível de discriminação entre as classes tornando possível analisar os agrupamentos das regiões de interesse na área de estudo como mostraram os mapas de classificação (Figura 3 e 5).

A adesão de ambos os métodos de classificação (Classificação Supervisionada e GEOBIA) utilizando a mineração de dados pela árvore de decisão para as imagens de satélite de média resolução do Landsat 8 Sensor $O L I$, apresentou resultados equivalentes às abordagens tradicionais para a área de estudo, que possui complexidade mesmo com a metodologia de GEOBIA utilizando mais informações para a classificação. Prudente et al. (2017) constataram que o processo de classificação de uso e cobertura da terra pelo método GEOBIA apresenta eficiência equivalente à análise pixel a pixel.

Efetivar os dois métodos para avaliar os níveis de classificação da cobertura da terra sendo o primeiro de Classificação Supervisionada e o segundo orientado a objeto (GEOBIA), permite a comparação estatística e visual entre os dois. Individualmente, os resultados foram bastante satisfatórios na abordagem de Classificação Supervisionada com $89 \%$, verificadas na matriz de confusão. Já para a abordagem da GEOBIA, o nível de Exatidão Global apresentou o maior índice, com 91\%. Ressalta-se, portanto, que ambas as classificações foram consideradas satisfatórias.

\section{CONCLUSÕES}

O sensoriamento remoto mostrou eficiência em pesquisas relacionadas ao uso e cobertura da terra. A associação desses dados com o uso de SIGs torna suas aplicações ainda mais abrangentes, trazendo uma pesquisa com menores custos monetários e de trabalho, possibilitando o acompanhamento em escalas de tempo maiores.

O índice Kappa auxiliou na descrição e teste do grau de concordância, com valor de 0,84 para a Classificação Supervisionada e 0,94 para a GEOBIA, resultado de qualidade excelente para ambos os métodos de classificação do uso da terra para o setor norte da Ilha do Maranhão, destacando a eficiência dos métodos utilizados.

A GEOBIA permitiu a partição de regiões (objetos), foi possível realizar a segmentação da área de estudo, encontrando os valores de escala e fusão que melhor representassem a separação dos mesmos. A técnica de mineração de dados por árvore de decisão facilitou a classificação da cobertura e uso da terra no método de GEOBIA, visto que, permitiu a obtenção dos principais parâmetros para a classificação da imagem. A vegetação de mangue e vegetação rasteira, que são bastante representativas, se distribuem majoritariamente, de maneira contínua, entre as classes: água e as áreas urbanas, mostrando que o intenso adensamento populacional evoluiu através do aterramento de mangues e desmatamento da vegetação nativa.

Novas abordagens em estudos futuros, considerando diferentes datas, sensores e algoritmos são necessárias para consolidar a discussão sobre o uso dessas metodologias, recomenda-se uma análise de uma comparação individual entre as classes definidas para a região. Os resultados do método de Classificação Supervisionada e do método de GEOBIA utilizando dados disponíveis de imagens de satélite, apesar de suas limitações, se mostraram satisfatórios conforme os valores de referência que os resultados apresentaram, o que torna interessante sua aplicação para iniciativas de mapeamento em outros municípios brasileiros, em especial na zona costeira. 


\section{REFERÊNCIAS}

ADDINK, E. \& KLEINHANS, M. Recognizingmeanders to reconstruct river dynamics of the Ganges. In: HAY, G., BLASCHKE, T., MARCEAU, D. (eds), GEOBIA (Pixels, objects, intelligence: geographic object based image analysis for the 21 st century) 48 , part $4 / \mathrm{C} 1$ of The international archives of the photogrammetry, remote sensing and spatial information sciences, Calgary: International Society for Photogrammetry and Remote Sensing (ISPRS). 2008.

BLASCHKE, T.; HAY, G.J.; KELLY, M.; LANG, S.; HOFMANN, P.; ADDINK, E; FEITOSA, R.Q.; MEER, F.V D.; WERFF, H.V.D.; COILLIE, F.V.; TIEDE, D. Geographic object-based image analysis-towards a new paradigm. ISPRS journal of photogrammetry and remote sensing, v. 87, p. 180-191, 2014.

CINTRÓN, G. \& SCHAEFFER-NOVELLI, Y. Ecology and management of New World mangroves. In: SEELIGER $\mathrm{U}(\mathrm{Ed})$, Coastal plant communities of Latin America Academic Press, San Diego, p. 233-258, 1992.

CONGALTON, R. \& GREEN, K. Assessing the Accuracy of Remotely Sensed Data: principles and practices. Boca Raton: CRC/Taylor \& Francis, 2009.

COUTO, E.; SILVEIRA F.L.; ROCHA, G. Marine biodiversity in Brazil: the current status. Gayana, v. 67, p. 327-340, 2003.

DE MATOS, W.B.; DOS SANTOS, G.M.C.; DA SILVA, U.E.B.; GONÇALVES, E. G. R.; DA SILVA, A.R. 2012. Paracoccidioidomycosis in the state of Maranhão, Brazil: geographical and clinical aspects. Revista da Sociedade Brasileira de Medicina Tropica, v. 45, p. 385-9, 2012.

EL-ROBRINI, M.; ALVES, M.A.M.S.; MARQUES JR., V.; ELROBRINI, M.H.S.; FEITOSA, A.C.; TAROUCO, J.E.F.; SANTOS, J.H.S.; VIANA, J.R. Maranhão. In: Dieter Muehe. (Org.). Erosão e Progradação no Litoral Brasileiro. Brasília: Ministério do Meio Ambiente, p. 87-130, 2006.

FABBRI, K.P. A methodology for supporting decision making in integrated coastal zone management. Ocean \& Coastal Management, v. 39, p. 51-62, 1998.

FEITOSA, A.C. Morfodinâmica da área costeira a nordeste da Ilha do Maranhão. In: SIMPÓSIO NACIONAL DE GEOMORFOLOGIA, Florianópolis. 1998. Anais... Florianópolis: Geosul, p. 252-255,

FEITOSA, A.C. \& TROVÃO, J. R. Atlas Escolar do Maranhão: espaço geo-histórico e cultural. João Pessoa: Grafset; 2006.

FRANCISCO, C.N. \& ALMEIDA, C.M. Avaliação de Desempenho de Atributos Estatísticos e Texturais em uma Classificação de Cobertura da Terra Baseada em Objeto. Boletim de Ciências Geodésicas, v. 18, n. 2, p. 302-326, 2012a.

FRANCISCO, C.N. \& ALMEIDA, C.M. Data Mining Techniques and Geobia Applied to Land Cover Mapping. In: Internacional Conference on Geographic Object-Based Image Analysis, 4. (GEOBIA), 2012, Rio de Janeiro. Proceedings...São José dos Campos: INPE, 2012b. p. 89-94. Disp. em: http://urlib.net/8JMKD3MGP8W/3BT2APS.

HAN, J. \& KAMBER, M. Data Mining: Concepts and Techniques. San Francisco: Morgan Kaufman Publisher, 2006, $743 \mathrm{p}$

HARALICK, R. \& SHANMUGAM, K. Computer classification of reservoir sandstones. IEEE Transactions on Geoscience Electronics, v. 11, n. 4, p. 171-177, out. 1973.

IBGE. Instituto Brasileiro de Geografia e Estatística Censo 2010. Disp. em: <www.ibge.gov.br/>. Acesso em: 20/setembro/2018.

IBGE - Instituto Brasileiro de Geografia e Estatística. Zoneamento Geoambiental do Estado do Maranhão. Brasília: IBGE, 1997.

JENSEN, J. R. Sensoriamento remoto do ambiente: uma perspectiva em recursos terrestres. Tradução: EPIPHANIO, J.C.N. São José dos Campos: Parêntese, 2009. 598 p. Tradução de Remote sensing of the environment: na Earth resource perspective.

KIM, M.; WARNER, T. A.; MADDEN, M.; ATKINSON, D.S Multi-scale GEOBIA with very high spatial resolution digital aerial imagery: scale, texture and image objects. International Journal of Remote Sensing, v. 32, n. 10, p. 2825-2850, 2011.

LANDIS, J.R. \& KOCH, G.G. The measurement of observer agreement for categorical data. Biometrics, v. 33, n. 01, p. 159174, 1977.

MACHADO, A.M.B. Comparação de Imagens OLI/LANDSAT8 E MSI/SENTINEL-2 no Mapeamento de Uso e Cobertura da Terra na Ilha do Maranhão. Revista Equador, v. 9, p. 209-232, 2020.

MOCHEL, F.R. \& PONZONI, F.J. Spectral characterization of mangrove leaves in the Brazilian Amazonian Coast: Turiaçu Bay, Maranhão State. Anais da Academia Brasileira de Ciências, v. 79, n. 4, p. 683-692, 2007.

NAVULUR, K. Multispectral image analysis using the objectoriented paradigm. Boca Raton, USA: Taylor \& Francis Group, 165 p., 2006.

NOVACK, T. Classificação da cobertura da terra e do uso do solo urbano utilizando o sistema InterIMAGE e imagens do sensor QuickBird. 2009. 214 p. (INPE-16594-TDI/1580). Dissertação (Mestrado em Sensoriamento Remoto) - Instituto Nacional de Pesquisas Espaciais, São José dos Campos, 2009. Disp. em: Acesso em: 01 out. 2018.

PEREIRA, T.D.J.F.; DE CASTRO, A.C.L.; FERREIRA, H.R.S.; SOARES, L.S.; SILVA, M.H.L.; DE JESUS AZEVEDO, J.W.; FRANÇA, V.L.; DOS SANTOS MOREIRA, M. Extrativismo De Mariscos Na Ilha Do Maranhão (MA): implicações ecológicas e socioeconômicas. Revista de Políticas Públicas, v. 21, n. 2, p. 831-854, 2018.

PINHO, C M.D. Análise orientada a objetos de imagens de satélite de alta resolução espacial aplicada à classificação de cobertura do solo no espaço intra-urbano: o caso de São José dos Campos - SP. São José dos Campos. 2005. Dissertação (Mestrado em Sensoriamento Remoto) - Instituto Nacional de Pesquisas Espaciais, São José dos Campos.

PIORSKI, G.M.R., GOMES, L.N., JUNIOR, J.R.P.; PIORSKI, N.M. Subsídios para o manejo da visitação na praia de Carimã, Raposa-MA. Caminhos de Geografia, v. 10, n. 32, 2009.

PRUDENTE, V.H.; SILVA, B.B.D.; JOHANN, J.A.; MERCANTE, E.; OLDONI, L.V. Comparative assessment between per-pixel and object-oriented for mapping land cover and use. Engenharia Agricola, v. 37, n. 5, p. 1015-1027, 2017.

RANGEL, M.E.S. Contribuição dos dados integrados dos sistemas sensores TM/ Landsat-5 e ERS-1/SAR para o estudo de uso e cobertura da terra no nordeste da Ilha do Maranhão. São José dos Campos. 2000. 112 p. Dissertação (Mestrado em Sensoriamento Remoto) - Instituto Nacional de Pesquisas Espaciais, São José dos Campos.

RIBEIRO, R.P.; ALVES, P.R.; ALMEIDA, Z.D.S.; RUTA, C. A new species of Paraonis and an annotated checklist of polychaetes from mangroves of the Brazilian Amazon Coast (Annelida, Paraonidae). ZooKeys, v. 740, n. 1-34, 2018.

RODRIGUES, T.C.S. Classificação da Cobertura e do Uso da Terra com Imagens Worldview-2 de Setores Norte da Ilha do Maranhão por Meio do Aplicativo Interimage e de Mineração de Dados. São José dos Campos. 2014. 87 p. Dissertação (Mestrado em Sensoriamento Remoto) - Instituto Nacional de Pesquisas Espaciais, São José dos Campos.

RWANGA, S.S. \& NDAMBUKI, J. M. Accuracy assessment of land use/land cover classification using remote sensing and GIS. International Journal of Geosciences, v. 8, n. 04, p. 611, 2017.

SOUSA, E.B; COSTA, V.B.; PEREIRA, L.C.C., COSTA R.M. Microfitoplâncton de águas costeiras amazônicas: ilha Canela (Bragança, PA, Brasil). Acta Botanica Brasilica, v. 22, p. 626-636, 2008. 
SUN, J.; YANG, J.; ZHANG, C.; YUN, W.; QU, J. Automatic remotely sensed image classification in a grid environment based on the maximum likelihood method. Mathematical and Computer Modelling, v. 58, n. 3, p. 573- 581, 2013.

VER, L.M.B.; MACKENZIE, F.T., LERMAN, A. Carbon cycle in the coastal zone: effects of global pertubations and change in the past three centuries. Chemical Geology, v. 159, p. 283-304, 1999.

VILELA, F.V.; SOARES, V.P.; ADUAN, R.E.; FERNANDES FILHO, E.I.; PEREIRA, R.D.A.; RIBEIRO, C.A.A.S. Uso de imagens orbitais como base de dados para projetos de reforma agrária. Revista Árvore, v. 29, n. 4, p. 627-638, 2005.
WALD, L.; RANCHIN, T.; MANGOLINI, M. Fusion of satellite images of different spatial resolutions: assessing the quality of resulting images. Photogrammetric Engineering \& Remote Sensing, v. 63, n. 6, p. 691-699, 1997.

Submetido em 16 de maio de 2019 Aceito para publicação em 10 de outubro de 2020 